\title{
Crises Financeiras e o Papel da Política Econômica: uma Abordagem Pós-Keynesiana
}

\section{Financial Crises and the Role of Economic Policy: a Post- Keynesian Approach}

\author{
Lucas Lautert Dezordi* \\ Marcelo Curado**
}

\begin{abstract}
Resumo: O objetivo central deste artigo consiste em discutir a condução das políticas econômicas, monetária e fiscal, em um ambiente de fragilidade financeira através de um modelo macrodinâmico. A evidência histórica destaca que, para evitar períodos de depressão econômica e pânicos prolongados, como ocorreram na Grande Depressão, as políticas econômicas devem ser coordenadas. A combinação de baixa taxa de juros e aumento do déficit público torna-se fundamental para estabilizar o sistema. O modelo proposto parte da identidade macroeconômica kaleckiana de determinação de lucro e, em seguida, utiliza os conceitos fundamentais do pensamento pós-keynesiano. Os resultados indicam que a combinação de queda na taxa de juros deve ser seguida por uma expansão do déficit público para estabilizar o sistema em períodos de severa fragilidade financeira das empresas. Em casos de colapso da eficiência marginal do capital, decorrente, por exemplo, de uma deterioração do estado de confiança, a queda dos juros no nível da "armadilha da liquidez" torna-se fundamental para estabilizar o modelo.
\end{abstract}

Palavras-chave: Fragilidade financeira. Políticas econômicas. Macroeconomia póskeynesiana.

\begin{abstract}
The current paper aims primarily discussing the conducting of economic, monetary and fiscal policy, during a time of financial fragility through a macro dynamic model. Historical evidences show that in order to avoid periods of economical depression and enduring panics as happened during the Great Depression, the economical policy should be coordinated. The combination of low interest rate and the increase of public deficit is essential to stabilize the system. The proposed model starts from the macroeconomic Kaleckiana identity of profit determination and in addition, it uses the fundamental concepts of the post-Keynesian thoughts. The results indicate that the combination of interest rate drop and expansion of the public deficit is relevant to stabilize the system in periods of financial vulnerability of private companies. In case of collapse of the marginal capital efficiency as a consequence of depreciation of the state trust, dropping interests to a "liquidity trap" level, it becomes crucial to stabilize the model.
\end{abstract}

Keywords: Financial fragility. Economic policy. Post Keynesian macroeconomics.

JEL Classification: E44.

\footnotetext{
* $\quad$ Doutor em Desenvolvimento Econômico pelo Programa de Pós-Graduação em Economia (PPGDE) da Universidade Federal do Paraná (UFPR). Professor da Universidade Positivo. E-mail: ldezordi@up.com.br

** Doutor em Economia pela Universidade Estadual de Campinas (Unicamp). Professor do Programa de Pós-Graduação em Economia (PPGDE) da Universidade Federal do Paraná (UFPR). E-mail: mlcurado@gmail.com
} 


\section{Introdução}

O presente artigo tem como objetivo principal discutir, a partir de um modelo macroeconômico dinâmico pós-keynesiano, ${ }^{1}$ a condução das políticas econômicas em um ambiente de fragilidade financeira. Destaca-se, assim, a necessidade de se utilizar e coordenar os instrumentos fiscais e monetários de forma a estabilizar o sistema econômico, como proposto por Minsky, na necessidade de se utilizar o Grande Governo e o Grande Banco para evitar períodos de depressão econômica e pânicos prolongados, como ocorreu na década de 1930.

Para uma discussão das crises financeiras, Charles Kindleberger torna-se uma referência de vital importância. Kindleberger e Aliber (2009) e Bordo (2003) argumentam, por meio de uma análise histórica do capitalismo, que as crises financeiras são eventos recorrentes e não isolados. Enfatizam de forma surpreendente que, de 1970 até o período atual, as volatilidades nos preços das ações, moedas, commodities, imóveis e ações estão cada vez mais severas e frequentes. ${ }^{2}$

Desde a Grande Depressão de 1929, a literatura de inspiração keynesiana tem argumentado em favor da utilização conjunta dos instrumentos fiscais e monetários para atenuar os efeitos das crises sobre o lado real da economia. ${ }^{3} \mathrm{O}$ argumento central de Minsky (1986), por exemplo, consiste em destacar que as fortes recessões dos anos de 1975 e 1982 nos Estados Unidos não se transformaram em uma depressão econômica graças à utilização de políticas macroeconômicas ativas. A atuação do Grande Governo, estabilizando a demanda agregada, melhorando o fluxo de caixa das empresas e permitindo a reestruturação de seus ativos, somada às ações do Federal Reserve System, em especial, à sua função de emprestador de última instância, tornaram-se fundamentais para evitar e minimizar os impactos mais profundos das crises financeiras sobre o lado real da economia.

A crise financeira de 2007-2008, iniciada no mercado imobiliário norte-americano, é um exemplo claro da implementação do receituário keynesiano em momentos de grave crise financeira. As evidências são claras em demonstrar que a crise foi amenizada com a combinação de estímulos monetários e crescimento

$1 \quad$ Para um estudo mais detalhado do pensamento pós-keynesiano, consultar Davidson (2002).

2 No século XIX, Kindleberger e Aliber (2009) citam 17 eventos relacionados a boom e crash, muitos deles, seguidos de pânico e corrida bancária, relacionados à inadimplência, ao ciclo de investimento nas ferrovias, nas hidrovias e na infraestrutura e ao preço das commodities. Bordo (2003) analisa o comportamento dos preços dos mercados acionários para um grupo de 19 países industrializados entre 1959 e 2003. O estudo revela a ocorrência de 52 quedas acentuadas e persistentes no mercado de ações. Todos os países analisados registraram ao menos uma queda importante e persistente no preço de suas ações. A existência de uma elevada correlação entre a deflação no preço dos ativos financeiros e a ocorrência de um processo recessivo é outro resultado destacado pelo estudo.

3 A rigor, Keynes (1996) foi o primeiro a prescrever o uso de políticas econômicas ativas em momentos de grande instabilidade, particularmente em períodos de colapso da eficiência marginal do capital e de elevação abrupta da preferência pela liquidez nos agentes. 
expressivo do déficit público. Em 2008, por exemplo, as despesas correntes do governo norte-americano aumentaram principalmente pela ampliação das despesas públicas e transferências. O déficit público foi de 7,8\% do PIB. O ano de 2009 foi marcado pela ampliação expressiva dos estímulos fiscais. O déficit público atingiu a marca recorde de 14,7\% do PIB, com forte ampliação das despesas correntes. Nos três anos seguintes, o resultado nominal do governo norte-americano foi um déficit na ordem de 10,0\% do PIB, isto é, fortemente expansionista. Ao mesmo tempo, o Federal Reserve atuou em duas frentes no campo monetário: reduziu a meta de taxa de juros de curto prazo para um nível entre $0-0,25 \%$ ao ano em final de 2008, mantendo-a por mais de quatro anos seguidos; e atuou como emprestador de última instância, evitando assim a falência de grandes corporações financeiras como, por exemplo, o Citibank. ${ }^{4}$

Nesse contexto, os questionamentos centrais deste artigo são: Como as políticas econômicas devem ser coordenadas? A redução da taxa de juros é suficiente para estabilizar a economia em períodos de crises econômicas?

Este artigo é organizado da seguinte forma. Após a introdução, a segunda seção apresenta a estrutura básica do modelo. Seu ponto de partida é o artigo de Taylor e O'Connell (1985), que formaliza a discussão de fragilidade financeira desenvolvida originalmente por Hyman Minsky. A terceira seção desenvolve o tema da fragilidade financeira para uma nação a partir dos trabalhos de Foley (2001) e Lima e Meirelles (2004a). A quarta seção apresenta os impactos da crise de confiança sobre o sistema. A quinta seção pretende, a partir do modelo teórico elaborado, compreender como as políticas econômicas devem ser conduzidas para estabilizar o sistema econômico em um ambiente de fragilidade. A última seção traz as considerações sobre as políticas econômicas e foram realizadas com base nos resultados obtidos do modelo, o qual considera que a instabilidade financeira da economia é causada por crises de dívida das empresas privadas e as crises bancária. Elas estão bem interligadas. Com isso, busca-se identificar como as políticas econômicas devem ser conduzidas nessas circunstâncias.

\section{Estrutura Básica do Modelo}

O ponto de partida do modelo é a identidade macroeconômica de Kalecki adaptada para uma economia aberta. ${ }^{5} \mathrm{~A}$ identidade demonstra que a taxa de lucro dos capitalistas é obtida pela soma dos investimentos das empresas, do déficit público, do saldo em transações correntes do balanço de pagamentos e do consumo dos capitalistas menos a poupança dos trabalhadores.

4 Dados do Bureau of Economic Analysis (BEA) e Fundo Monetário Internacional (FMI).

5 Para uma discussão detalhada sobre o tema, veja, entre outros, Wray (2006). 


$$
Y_{\pi}=I+(G-T)+(X-M)+C_{\pi}-S_{W}
$$

em que:

$Y_{\pi}=$ lucro bruto após o pagamento de impostos;

$I$ = investimento das empresas;

$(G-T)=$ déficit do governo; ${ }^{6}$

$(X-M)$ = saldo em transações correntes do balanço de pagamentos;

$C_{\pi}=$ consumo dos capitalistas; $\mathrm{e}$

$S_{W}=$ poupança dos trabalhadores.

Considerando uma economia aberta, com o setor público e trabalhadores gastando toda sua renda $\left(S_{W}=0\right)$, o lucro agregado será determinado por:

$$
\mathrm{Y}_{\pi}=I+(G-T)+(X-M)
$$

A função consumo dos capitalistas é descrita por: $\mathrm{C}_{\pi}=\mathrm{cY}_{\pi}$ e $0<c<1$, em que $c$ representa a propensão marginal a consumir do lucro realizado. Pode-se deduzir, portanto, que:

$$
\begin{aligned}
& Y_{\pi}-c Y_{\pi}=I+(G-T)+(X-M) \\
& (1-c) Y_{\pi}=I+(G-T)+(X-M)
\end{aligned}
$$

Como a taxa de poupança sobre os lucros é dada por: $s=1-c$, a identidade pode ser expressa pelas relações das contas nacionais, conforme a equação 3. Esta indica que a soma das poupanças privada (capitalista) com a externa ${ }^{7}$ é igual à soma do investimento nacional com o valor do déficit público.

$$
s Y_{\pi}+(M-X)=I+(G-T)
$$

Dividindo-se ambos os lados da equação 3 pelo estoque de capital $K$, obtêm-se:

$$
s r+d=g+b
$$

$6 \quad$ Pelas contas nacionais, as receitas correntes menos as despesas correntes representam o saldo do governo em conta corrente (poupança do governo, $S_{g}$ ). Esse modelo, por simplificação, considera que o déficit do governo é igual ao saldo do governo em conta corrente com sinal trocado $\left(-S_{g}\right)$. Ou seja, se há déficit público, então, necessariamente, $S_{g}<0$ e $G>T$.

7 A poupança externa, na identidade macroeconômica básica, é igual ao déficit em transações correntes do balanço de pagamentos ou igual ao superávit da conta financeira. 
em que:

$$
r=\frac{Y_{\pi}}{K} ; \quad d=\frac{(M-X)}{K} ; \quad g=\frac{I}{K} ; \quad b=\frac{(G-T)}{K}
$$

A soma da poupança privada dos capitalistas em relação ao estoque de capital (sr) com o superávit da conta financeira do balanço de pagamentos em relação ao estoque de capital $(d)$ é igual à soma do investimento nacional sobre o estoque de capital (g), acrescido do déficit público em relação ao estoque de capital (b). ${ }^{8}$

O setor externo será estudado a partir do comportamento da conta financeira do balanço de pagamentos, tal como proposto por Foley (2001). Assume-se, por hipótese, a existência de uma relação direta entre as taxas de lucro ( $r$ ), a taxa de juros (i) e o resultado da conta financeira do balanço de pagamentos. Essas hipóteses são formalmente apresentadas na equação 5:

$$
d=d_{i} i+d_{r} r
$$

em que: $d_{i}, d_{r}>0$ e representam a sensibilidade da conta financeira do balanço de pagamentos em relação à taxa de juros e a taxa de lucro, respectivamente.

A relação positiva entre as variáveis (lucros e juros) e o resultado da conta financeira do balanço de pagamentos é justificada da seguinte forma: quanto maior a taxa de lucro ( $r$ ) da economia, maior a entrada de capitais produtivos (na forma de investimentos diretos) no país; por seu turno, quanto maior a taxa de juros doméstica (i) maior a entrada de capitais de curto prazo para o país.

Em síntese, taxas de juros e de lucros maiores tendem a gerar um superávit na conta financeira do balanço de pagamentos. O efeito de cada uma das variáveis sobre a conta financeira dependerá dos parâmetros positivos $d_{i}$ e $d_{r}$.

A equação da demanda por investimento, conforme a equação 6, descreve o comportamento do processo de acumulação de capital, g, que está diretamente relacionado à taxa de lucro $(r)$ e ao estado de confiança $(\rho)^{9}$ e indiretamente à taxa de juros, i. Essa equação foi sugerida originalmente por Taylor e O'Connell (1985).${ }^{10}$ A ideia central consiste em que se o preço da demanda $(r+\rho)$ for maior que o preço da oferta (i) dos bens de consumo de capital, ${ }^{11}$ ou seja, quando a eficiência marginal do capital - que é o valor presente dos lucros que se espera

8 Utilizam-se os termos superávit da conta financeira e déficit público para definir as razões $d$ e $b$, respectivamente, pois se seguem os padrões utilizados nas referências de análise, por Foley (2001). Entretanto, a interpretação delas deve considerar a possibilidade de déficit da conta financeira para $d<0$ e superávit orçamentário para $b<0$.

9 Taxa de lucro esperada no futuro.

10 Por medida de simplificação, omitiu-se a taxa de acumulação do capital autônoma, $\mathrm{g}_{0}$.

11 Por hipótese, a economia produz apenas um bem, portanto, o preço dos bens de capital é igual ao preço dos bens de consumo. 
obter no futuro em decorrência da aquisição do capital — for maior que a taxa de juros do mercado, maior será o volume de novos investimentos, de acordo com o parâmetro $h$.

$$
g=h(r+\rho-i)
$$

em que: $h>0$

Assume-se que o déficit público em relação ao estoque de capital é uma variável autônoma, determinada pela equação $7,{ }^{12}$ ou seja:

$$
b=b_{0}
$$

Substituindo as equações 5, 6 e 7 em 4, e resolvendo para a relação taxa de lucro-capital:

$$
r=\frac{h \rho-\phi i+b_{0}}{z}
$$

em que:

$z=\left(s+d_{r}-h\right)$ e necessariamente $s+d_{r}>h$.

$\phi=\left(h+d_{i}\right)$

Obtendo as derivadas:

$$
\frac{\partial r}{\partial i}=-\frac{\phi}{z} ; \quad \frac{\partial r}{\partial \rho}=\frac{h}{z} ; \quad \frac{\partial r}{\partial b_{0}}=\frac{1}{z}
$$

A derivada do lucro em relação à taxa de juros é negativa. A magnitude dessa relação depende dos parâmetros: $s, d_{r}$, $h$ e $d_{i}$. Quanto menores forem os parâmetros $\left(s\right.$ e $\left.d_{r}\right)$ e maiores forem os parâmetros ( $h$ e $\left.d_{i}\right)$, maior será o valor, em módulo, da derivada do lucro em relação à taxa de juros.

A relação entre o estado de confiança e a taxa de lucro é expressa pela derivada $\partial \mathfrak{r} / \partial \rho=\mathrm{h} /$. Seu valor é positivo e depende dos parâmetros $s, d_{r}$ e $h$. Quanto maiores forem os valores dos parâmetros ( $\left(s\right.$ e $\left.d_{r}\right)$, menor será o impacto do estado de confiança dos agentes econômicos sobre a taxa de lucro. Por outro lado, quanto maior for o valor do parâmetro (h), maior será o impacto de $\rho$ na renda dos

12 Destaca-se que, para manter constante essa razão, o déficit público (G-T) deve aumentar a uma taxa igual ao crescimento do estoque de capital, $K$ 
capitalistas. Ressalta-se que a análise das derivadas deve respeitar a condição de estabilidade do modelo $\left(s+d_{r}\right)>h$.

Consequentemente, tem-se que o valor, em módulo, da derivada do lucro em relação à taxa de juros é sempre maior que a derivada do lucro em relação ao estado de confiança: $|\partial \mathrm{r} / \partial \mathrm{i}|>|\partial \mathrm{r} / \partial \rho|$. A derivada do lucro em relação ao déficit público/estoque de capital é positiva. Quanto menores os parâmetros $\left(s\right.$ e $\left.d_{r}\right)$ e maior $h$, maior será o valor dessa derivada. Sendo $\phi<1$, então $|\partial \mathrm{r} / \partial \mathrm{i}|<\left|\partial \mathrm{r} / \partial \mathrm{b}_{0}\right|$.

Substituindo a equação 8 na equação 6 , pode-se obter o processo de acumulação do capital como função das seguintes variáveis:

$$
g=\frac{h(h+z)}{z} \rho-\frac{h\left(h+d_{i}+z\right)}{z} i+\frac{h}{z} b_{0}
$$

Obtendo-se as derivadas parciais em relação à acumulação de capital: ${ }^{13}$

$$
\frac{\partial g}{\partial \rho}=\frac{h(h+z)}{z} ; \quad \frac{\partial g}{\partial i}=-\frac{h\left(h+d_{i}+z\right)}{z} ; \frac{\partial g}{\partial b_{0}}=\frac{h}{z}
$$

Assim, uma melhora no estado de confiança dos agentes econômicos tem um impacto positivo sobre a taxa de crescimento da acumulação de capital. Sua derivada depende dos parâmetros $s, d_{r}$ e $h$. Como $\partial g / \partial \rho=h^{2} / z+h$. Um aumento nos parâmetros $\left(s\right.$ e $d_{r}$ ) reduzirá os efeitos de $\rho$ na taxa de crescimento da acumulação de capital. Por seu turno, quanto maior for o parâmetro $h$, maior será o valor dessa derivada.

A derivada da taxa de crescimento do estoque de capital em relação à taxa de juros é negativa. A magnitude dessa relação depende dos parâmetros $s, d_{r}$ h e $d_{i}$. Quanto maiores os parâmetros ( $s$ e $d_{r}$ ), menor a eficiência dos juros sobre a taxa de acumulação de capital. Quanto maiores os parâmetros (h e $d_{i}$ ), maior a eficiência da política monetária. Em valores absolutos, o impacto da taxa de juros sobre g é sempre maior que o do estado de confiança $|\partial g / \partial \mathrm{i}|>|\partial g / \partial \rho|$. Por sua vez, a derivada da taxa de acumulação de capital em relação ao déficit público/estoque de capital é positiva. Assim, quanto menores forem os parâmetros ( $s$ e $d_{r}$ ) e maior o valor de $h$, maior será essa derivada. Tendo em vista que a soma dos parâmetros $\left(d_{i}+s+d_{r}\right)<1$, então, tem-se que $|\partial \mathrm{g} / \partial \mathrm{i}|<\left|\partial \mathrm{g} / \partial \mathrm{b}_{0}\right|$.

Apresentada a estrutura básica do modelo, o passo seguinte diz respeito à construção das fronteiras de endividamento, elemento discutido na próxima seção.

13 As derivadas da taxa de acumulação de capital com as variáveis podem ser expressas também pelas seguintes relações: $\frac{\partial g}{\partial \rho}=\frac{h\left(s+d_{r}\right)}{z} \mathrm{e} \frac{\partial g}{\partial i}=\frac{-h\left(d_{i}+s+d_{r}\right)}{z}$ 


\section{Fronteiras de Endividamento: Hedge, Especulativa e Ponzi}

Foley (2001) e Lima e Meirelles (2004a) descrevem o fluxo de caixa das empresas em termos agregados pela seguinte identidade de recursos e usos:

$$
R+B \equiv I+F
$$

A equação 10 informa que as receitas operacionais líquidas, $R$, mais os novos empréstimos, $B$, são iguais aos fundos para investimentos, $I$, mais os serviços da dívida, $F$.

A mudança na dívida ao longo do tempo $\partial \mathrm{D} / \partial t$ é dada por:

$$
\dot{D}=B=I+F-R
$$

Considerando que o serviço da dívida pode ser expresso por $F=i D$, o investimento como proporção do estoque de capital (ativo das empresas) no período $t$, como $\mathrm{g}=I K$, e $r=R K$, a mudança na dívida pode ser descrita por:

$$
\dot{D}=(g-r) K+i D
$$

Lima e Meirelles (2004a), a partir da classificação originalmente desenvolvida por Minsky (1986), definem a situação financeira das empresas privadas por: ${ }^{14}$

Hedge $(\mathrm{H}): R \geq I+F$ ou $B \leq 0$

Especulativo (S): $R<I+F$ ou $I>B>0$

Ponzi (P): $R \leq F$ ou $B \geq I$

Dividindo ambos os lados pelo estoque de capital (ativo das empresas) no período $t$, obtêm-se:

$$
\frac{\dot{D}}{K}=(g-r)+i \frac{D}{K}
$$

Em uma análise de estática comparativa $\dot{D} K=0$. A relação dívida sobre o estoque de capital é dada por $\delta=\mathrm{D} / \mathrm{K}$, com isso:

$$
r-i \delta=g
$$

14 Relações apresentadas por Lima e Meirelles (2004a, p. 7). 
A ideia geral de utilizar a equação 14 é justificada da seguinte forma. Se a economia, em seu agregado, apresenta uma situação em que a receita operacional líquida sobre o ativo, descontada dos serviços da dívida-capital, é maior que o gasto com investimento sobre capital, então podemos entender que esta é uma economia hedge e, portanto, $\mathrm{r}-\mathrm{i} \delta>\mathrm{g}$.

Se por outro lado, devido ao período de expansão econômica e da necessidade de se aumentar o volume de investimentos, as empresas em seu agregado apresentam um fluxo de caixa no qual a receita operacional líquida sobre o ativo, descontada dos serviços da dívida-capital, é menor que o gasto com investimento sobre capital então sua situação é definida como especulativa e: $r-i \delta<$ g sendo r-i $>>0$.

Seguindo o mesmo raciocínio, pode-se concluir que uma situação em que $\mathrm{r}$-i $\delta<$ g e r-i $\delta<0$ representa uma economia Ponzi. Nessa situação, os lucros das firmas não cobrem o pagamento dos serviços de sua dívida. As firmas precisam tomar recursos emprestados no mercado com o objetivo de honrar suas obrigações.

Tabela 1 - Fronteiras dos Regimes Financeiros de Minsky

\begin{tabular}{l|ll}
\hline Regime financeiro & Relação & Fronteira \\
\hline Hedge $(\mathrm{H})$ & $\mathrm{r}-\mathrm{i} \delta \geq \mathrm{g}$ & \\
Especulativo $(\mathrm{S})$ & $\mathrm{r}-\mathrm{i} \delta<\mathrm{g}$ & $\mathrm{r}-\mathrm{i} \delta_{(\mathrm{h}-\mathrm{s})}=\mathrm{g}$ \\
Ponzi (P) & $\mathrm{r}-\mathrm{i} \delta \leq 0$ & $\mathrm{r}=\mathrm{i} \delta_{(\mathrm{s}-\mathrm{p})}$ \\
\hline
\end{tabular}

Fonte: Elaboração própria com base em Lima e Meirelles (2004a, p. 7).

A partir deste ponto, pretende-se analisar como o estado de confiança e a condução da política monetária e fiscal podem afetar as fronteiras de endividamento e, com isso, reduzir/ampliar os espaços entre as posições financeiras propostas originalmente por Minsky (1986) e adaptadas por Lima e Meirelles (2004a).

\section{I Fronteira da relação Dívida sobre Estoque da Capital: Hedge-Especulativa $\left(\delta_{\mathrm{h}-\mathrm{s}}\right)$.}

Utilizando as equações da taxa de lucro (8) e da taxa de acumulação de capital (9) e isolando a relação dívida sobre o capital das empresas na fronteira entre uma situação hedge para especulativa, obtém-se a equação 15.

$$
\begin{gathered}
i \delta_{h-s}=r-g \\
\delta_{h-s}=\frac{1}{i}\left[\frac{h\left(1-s-d_{r}\right)}{z} \rho+\left(\frac{1-h}{z}\right) b_{0}\right]+\left[\frac{h\left(s+d_{r}+d_{i}\right)-\left(h+d_{i}\right)}{z}\right]
\end{gathered}
$$


Alternativamente, escrevendo em função das derivas:

$$
\delta_{h-s}=\frac{1}{i}\left\{\left[\frac{\partial r}{\partial \rho}-\frac{\partial g}{\partial \rho}\right] \rho+\left[\frac{\partial r}{\partial b_{0}}-\frac{\partial g}{\partial b_{0}}\right] b_{0}\right\}+\left[\frac{\partial r}{\partial i}-\frac{\partial g}{\partial i}\right]
$$

A equação 16 indica que quanto maior a derivada do lucro em relação ao estado de confiança em relação à derivada da taxa de crescimento da acumulação de capital também sobre o estado de confiança, maior será a fronteira hedge-especulativa da dívida sobre capital. Desde que $\left(s+d_{r}\right)<1$, então pelas equações 8 e 9 , conclui-se que $|\partial r / \partial \rho|>|\partial g / \partial \rho|$ e, portanto, um aumento do estado de confiança $(\uparrow \rho)$ deve ampliar a fronteira hedge-especulativa $\left(\uparrow \delta_{\mathrm{h}-\mathrm{s}}\right){ }^{15}$

Um aumento autônomo do déficit público em proporção do estoque de capital $\left(\uparrow b_{0}\right)$ irá necessariamente ampliar a fronteira hedge-especulativa $\left(\uparrow \delta_{\mathrm{h}-\mathrm{s}}\right)$ visto que $h<1$ e $\left|\partial r / \partial b_{0}\right|>\left|\partial g / \partial b_{0}\right|$. As relações inversas entre a taxa de juros e as variáveis endógenas, lucro e crescimento do capital, devem ser analisadas pelas derivadas parciais. Como $\left(\mathrm{s}+\mathrm{d}_{\mathrm{r}}+\mathrm{d}_{\mathrm{i}}\right)<1$, tem-se como consequência $|\partial \mathrm{r} / \partial \mathrm{i}|>|\partial \mathrm{g} / \partial \mathrm{i}|$. Esse é um resultado importante, pois indica que o impacto de uma política monetária contracionista $(\uparrow i)$ sobre a taxa de lucro será maior do que sobre o crescimento da acumulação de capital: $|\partial \mathrm{r} / \partial \mathrm{i}|>|\partial \mathrm{g} / \partial \mathrm{i}|$, e a fronteira hedge-especulativa diminuirá $\left(\downarrow \delta_{\mathrm{h}-\mathrm{s}}\right)$.

\subsection{Fronteira da relação Dívida sobre Estoque de Capital: Especulativa-Ponzi $\left(\delta_{s p p}\right)$.}

Para determinar a fronteira Especulativa-Ponzi deve-se utilizar a fronteira determinada pela Tabela 1 e a pela equação 8. De tal forma que:

$$
\begin{gathered}
i \delta_{s-p}=r \\
\delta_{s-p}=\frac{1}{i}\left\{\left[\frac{h}{z}\right] \rho+\left[\frac{1}{z}\right] b_{0}\right\}-\frac{\phi}{z}
\end{gathered}
$$

Alternativamente, escrevendo em função das derivadas:

$$
\delta_{s-p}=\frac{1}{i}\left\{\left[\frac{\partial r}{\partial \rho}\right] \rho+\left[\frac{\partial r}{\partial b_{0}}\right] b_{0}\right\}+\frac{\partial r}{\partial i}
$$

A análise da fronteira especulativa-Ponzi ${ }^{16}$ é bem mais direta. Uma melhora no estado de confiança $(\uparrow \rho)$ expandirá a taxa de lucro $(\uparrow r)$ e ampliará a fronteira

\footnotetext{
15 Faz sentido econômico supor que a soma das propensões marginais a poupar e a importar seja menor do que a unidade.

16 Para uma discussão mais detalhada e específica sobre as condições financeiras das unidades pro-
} 
de fragilidade financeira $\left(\uparrow \delta_{\text {s-p }}\right)$. Mantendo-se constante o estado de confiança, um aumento na taxa de juros $(\uparrow i)$ reduzirá a taxa de lucro $(\downarrow r)$, tornando a fronteira da razão dívida sobre ativo em situação de crise financeira menor $\left(\downarrow \delta_{\text {s.p }}\right)$. Nessa situação, uma política fiscal expansionista $\left(\uparrow b_{0}\right)$ aumentará a taxa de lucro $(\uparrow r)$ e ampliará a fronteira especulativa-Ponzi $\left(\uparrow \delta_{\text {s-p }}\right)$. As equações indicam também uma relação direta entre juros e dívida-estoque de capital, pois as equações recíprocas descrevem uma relação não linear entre essas variáveis. Em síntese, os resultados obtidos até o momento permitem formalmente concluir que a má condução da política monetária pode agravar muito a situação financeira das empresas em nível agregado e, consequentemente, aprofundar à recessão.

\section{Análise Estática Comparativa das Fronteiras Financeiras}

Como descrito na Figura 1, as fronteiras são relações não lineares entre a dívida sobre o estoque de capital e a taxa de juros. Portanto, para uma taxa de juros, por exemplo, em torno de $10 \%(0,10)$, a curva gerada pela equação (15) é mais inclinada e menos sensível às variações nos juros do que a curva gerada pela equação (17). Com isso, se a economia, em seu agregado, encontra-se em uma fronteira especulativa-Ponzi, mudanças na taxa de juros terão mais efeitos na relação dívida-capital do que em uma fronteira hedge-especulativa.

Figura 1 - Regiões financeiras de Minsky

$\delta$

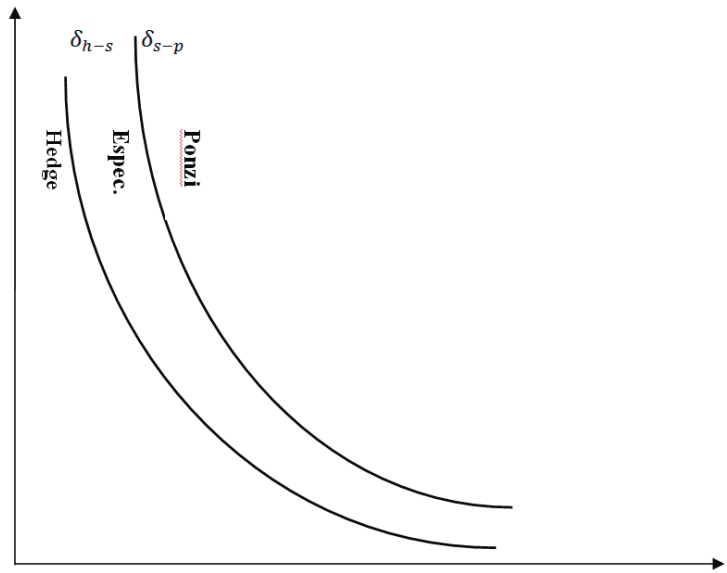

Fonte: Elaboração própria.

dutoras e sua dinâmica ao longo do tempo, ver Vercelli (1999) e Sordi e Vercelli (2003).

DEZORDI, L. L.; CURADO, M. Crises Financeiras e o Papel da Polífica Econômica... 
Conforme argumentado por Keynes no capítulo 12 da Teoria Geral, o estado das expectativas de longo prazo, crucial para as decisões de investimentos, não depende exclusivamente das probabilidades mensuráveis que se podem formular. Depende, de fato, crucialmente do estado de confiança. Uma crise financeira no sistema capitalista pode ser desencadeada por uma quebra das expectativas de longo prazo, ou seja, por uma deterioração do estado de confiança.

A Figura 2 ilustra o que ocorre com as curvas das fronteiras financeiras para um caso em que a economia apresente uma "quebra" nas expectativas de longo prazo: $(\downarrow \rho)$. Considerando inicialmente a equação 16 e sendo $\left(s+d_{r}\right)<1$, então pelas equações 8 e 9 , argumenta-se que $|\partial r / \partial \rho|>|\partial g / \partial \rho|$. Assim, uma queda no estado de confiança reduzirá a fronteira hedge-especulativa $\left(\downarrow \delta_{\mathrm{h}-\mathrm{s}}\right)$ e também a fronteira especulativa-Ponzi ( $\downarrow \delta_{\text {s-p }}$ ).

A região Ponzi fica maior e a região hedge mais estreita. Portanto, uma redução do estado de confiança - para uma mesma taxa de juros e relação dívida sobre estoque de capital - leva muitas empresas em situação hedge para posições financeiras especulativas e de situações especulativas para posições Ponzi, ou seja, desencadeia um processo de fragilização da estrutura financeira da economia. Esse processo é ilustrado pela Figura 2. Nessa situação, a economia em termos agregados fica mais susceptível à crise da dívida.

Figura 2 - Crise de confiança e situação financeira das empresas $\delta$

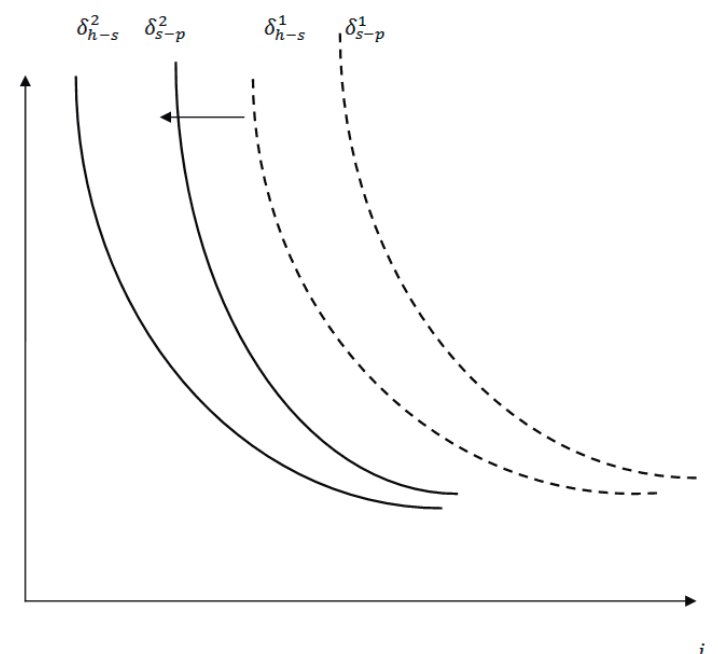

Fonte: Elaboração própria.

A relação entre o instrumental elaborado e o papel das políticas econômicas em um contexto de fragilização das posições financeiras da economia é clara. Em 
uma análise estática. pode-se, por exemplo, argumentar que a política fiscal expansionista de aumento da razão déficit público sobre o estoque de capital da economia deslocará as curvas da fronteira hedge-especulativa $\left(\uparrow \delta_{\mathrm{h}-\mathrm{s}}\right)$ e também a fronteira especulativa-Ponzi $\left(\uparrow \delta_{\text {s-p }}\right)$ para a direita, amenizando o colapso da eficiência marginal do capital e a crise financeira. Na seção seguinte, pretende-se aprofundar essa discussão da condução das políticas econômicas em momentos de crise.

\section{A Dinâmica Macroeconômica em Ambiente de Fragilidade Financeira}

Nesta seção, busca-se discutir a condução das políticas fiscal e monetária em um ambiente de fragilidade financeira. Apresentam-se duas equações diferenciais: uma para a taxa de juros ao longo do tempo e outra para o comportamento da razão déficit público sobre o estoque de capital, também ao longo do tempo. Uma crise financeira de dívida ocorrerá somente quando a economia estiver operando sobre a fronteira especulativa-Ponzi com $\mathrm{r}=\mathrm{i} \delta$ ou na área Ponzi, isto é, $\mathrm{r}<\mathrm{i} \delta$. Sua fronteira pode ser afetada, como demonstrado na seção anterior, pelo colapso da eficiência marginal do capital, que, nesse caso, amplifica a fragilidade financeira das empresas.

Minsky (1986) sugere algumas regras para prevenir ou amenizar uma crise financeira: i) intervenções bem calibradas dos bancos centrais, com sua função de emprestador de última instância (reduzindo os juros), evitando o processo de debt-deflation; ${ }^{17}$ e ii) ampliação do déficit público contracíclico, com o objetivo de sustentar a taxa de lucro agregada da economia. As regras de condução para a política monetária e fiscal apresentadas a seguir seguem, em seu espírito, a proposta de Minsky (1986):

a) Regra para a política monetária em ambiente de crise financeira:

$$
\frac{\partial i}{\partial t}=\Psi(g-\bar{g})+\theta\left(\delta_{s-p}\right)
$$

A equação dinâmica 19 indica uma atuação anticíclica na condução da política monetária, pois se a taxa de crescimento da acumulação de capital observada for menor do que a desejada pelos formuladores de política $(\mathrm{g}<\bar{g})$, então, a taxa de juros deverá cair de acordo com o parâmetro $\Psi$, que representa a velocidade de ajuste da política monetária (valor positivo e constante). Em uma crise financeira ou ampliação da fragilidade, a política monetária deverá ser flexibilizada. Por exemplo, em uma situação na qual a deterioração do estado de confiança reduz significativamente a fronteira da dívida especulativa-Ponzi, a autoridade monetária

17 Processo descrito em um artigo clássico em 1933, no fundo do poço da Grande Depressão, pelo economista norte-americano Irving Fisher, o qual destacou 49 "crenças" baseadas em observações, visões pessoais e pela própria experiência sobre booms e depressões. 
deve reduzir a taxa de juros para melhorar o fluxo de caixa das empresas, de acordo com o parâmetro de velocidade de ajuste $\theta$.

b) Dinâmica do déficit público em ambiente de crise financeira:

$$
\frac{\partial b}{\partial t}=-\beta(r-\bar{r})-\gamma\left(\delta_{s-p}\right)
$$

A política fiscal ao longo do tempo, expressa pela equação 20, mantém seu caráter anticíclico; quando a taxa de lucro sobe para um patamar acima da taxa de lucro de longo prazo, $\bar{r}$, então, o déficit público cai de acordo com o parâmetro de ajuste $\beta$. O déficit público também reage ao período de fragilidade financeira das empresas, cooperando com a iniciativa privada. Em uma situação de diminuição da fronteira especulativa-Ponzi, em virtude de uma deterioração do estado de confiança, o déficit público/estoque de capital sobe, dado parâmetro de velocidade de ajuste, $\gamma$. Pela identidade 2 fica evidente que uma atuação esperada do Estado em períodos de crise financeira seria a ampliação do déficit público para contribuir, entre outros elementos, com a expansão do lucro das empresas. ${ }^{18}$

Utilizando as relações em 8, 9 e 17, nas equações dinâmicas, podem ser escritas da seguinte forma, sendo $\mathrm{i}^{*}=\partial \mathrm{V} / \partial \mathrm{t} ; \dot{b}=\partial \mathrm{b} / \partial \mathrm{t}$ :

$$
\begin{aligned}
& i^{*}=\Psi\left[\frac{h(h-z)}{z}\right] \rho+\frac{1}{i} \theta\left(\frac{h}{z}\right) \rho-\Psi\left[\frac{h\left(h+d_{i}+z\right)}{z}\right] i+\Psi\left(\frac{h}{z}\right) b_{0}+\frac{1}{i} \theta\left(\frac{1}{z}\right) b_{0}-\Psi \bar{g}-\frac{\phi}{z} \\
& \dot{b}=-\frac{1}{i} \gamma\left(\frac{h}{z}\right) \rho-\beta\left(\frac{h}{z}\right) \rho+\beta\left(\frac{\phi}{z}\right) i-\frac{1}{i} \gamma\left(\frac{1}{z}\right) b_{0}-\beta\left(\frac{1}{z}\right) b_{0}+\beta \bar{r}+\gamma\left(\frac{\phi}{z}\right)
\end{aligned}
$$

Com isso, a matriz jacobiana será determinada pelas seguintes derivadas parciais:

$$
\begin{aligned}
& J_{11}=\frac{\partial i^{*}}{\partial i}=-\frac{1}{i^{2}} \theta\left[\frac{\partial r}{\partial \rho} \rho+\frac{\partial r}{\partial b_{0}} b_{0}\right]+\Psi\left[\frac{\partial g}{\partial i}\right] \\
& J_{12}=\frac{\partial i^{*}}{\partial b_{0}}=\frac{1}{i} \theta\left[\frac{\partial r}{\partial b_{0}}\right]+\Psi\left[\frac{\partial r}{\partial \rho}\right] \\
& J_{21}=\frac{\partial \dot{b}}{\partial i}=\frac{1}{i^{2}} \gamma\left[\frac{\partial r}{\partial \rho} \rho+\frac{\partial r}{\partial b_{0}} b_{0}\right]+\beta \frac{\partial r}{\partial i} \\
& J_{22}=\frac{\partial \dot{b}}{\partial b_{0}}=-\frac{1}{i} \gamma\left[\frac{\partial r}{\partial b_{0}}\right]-\beta\left[\frac{\partial r}{\partial b_{0}}\right]
\end{aligned}
$$

18 A limitação do crescimento das despesas públicas está relacionada à dinâmica da razão dívida pública líquida em relação ao PIB. O setor público também pode apresentar uma situação Ponzi, de fragilidade financeira. Contudo, o modelo em questão, não considera tal situação e, nesse sentido, a política fiscal anticíclica pode ser utilizada, se for o caso, para estabilizar a economia em períodos de severa fragilidade financeira das empresas privadas em nível agregado. 
A matriz jacobiana $J$ pode ser escrita na seguinte forma matricial:

$$
\left[\begin{array}{c}
\frac{\partial i}{\partial t} \\
\frac{\partial b}{\partial t}
\end{array}\right]=\left[\begin{array}{rc}
-\frac{1}{i^{2}} \theta\left[\frac{\partial r}{\partial \rho} \rho+\frac{\partial r}{\partial b_{0}} b_{0}\right]+\Psi\left[\frac{\partial g}{\partial i}\right] & \frac{1}{i} \theta\left[\frac{\partial r}{\partial b_{0}}\right]+\Psi\left[\frac{\partial r}{\partial \rho}\right] \\
\frac{1}{i^{2}} \gamma\left[\frac{\partial r}{\partial \rho} \rho+\frac{\partial r}{\partial b_{0}} b_{0}\right]-\beta \frac{\partial r}{\partial i} & -\frac{1}{i} \gamma\left[\frac{\partial r}{\partial b_{0}}\right]-\beta\left[\frac{\partial r}{\partial b_{0}}\right]
\end{array}\right] \cdot\left[\begin{array}{c}
(i-\vec{\imath}) \\
\vdots \\
(b-\bar{b})
\end{array}\right]
$$

Como as derivadas do lucro em relação ao estado de confiança e do déficit público/estoque de capital são positivas e a derivada da taxa de acumulação de capital sobre a taxa de juros é negativa, então necessariamente $\mathrm{J}_{11}<0$ e consequentemente o segundo termo da matriz é positivo: $\mathrm{J}_{12}>0$. Como a derivada do lucro em relação à taxa de juros é negativa, isto é, $\mathrm{J}_{21}>0$, o último termo da matriz terá valor negativo, isto é, $\mathrm{J}_{22}<0$.

Determinando a equação característica a partir da matriz jacobiana | J$\lambda I \mid=0$, gera-se o polinômio:

$$
\alpha_{0} \lambda^{2}+\alpha_{1} \lambda+\alpha_{2}=0
$$

A condição de estabilidade do sistema será dada por:

$$
\alpha_{0}>0 ; \alpha_{1}=-\left(a_{11}+a_{22}\right)>0 \therefore\left(a_{11}+a_{22}\right)<0 ; \quad \alpha_{2}=\left(a_{11} a_{22}\right)-\left(a_{12} a_{21}\right)>0
$$

A primeira condição é atendida, pois $\alpha_{0}=1$. A segunda condição depende do valor do traço da matriz jacobiana, o qual deverá ser negativo. A equação 29 indica que a soma de $\mathrm{J}_{11}$ com $\mathrm{J}_{22}$ é um valor necessariamente negativo: traço $|\mathrm{J}|<0$.

$$
\left(-\frac{1}{i^{2}} \theta\left[\frac{\partial r}{\partial \rho} \rho+\frac{\partial r}{\partial b_{0}} b_{0}\right]+\Psi\left[\frac{\partial g}{\partial i}\right]\right)+\left(-\frac{1}{i} \gamma\left[\frac{\partial r}{\partial b_{0}}\right]-\beta\left[\frac{\partial r}{\partial b_{0}}\right]\right)<0
$$

Para que $\alpha_{2}>0$, o determinante da matriz jacobiana deve ser necessariamente positivo. A equação 30 impõe essa condição por:

$$
\left\{\frac{1}{i^{2}}\left(\frac{\partial r}{\partial \rho} \rho+\frac{\partial r}{\partial b_{0}} b_{0}\right)(\theta \beta-\gamma \Psi h)+\frac{1}{i}\left(\gamma \Psi\left|\frac{\partial g}{\partial i}\right|-\theta \beta\left|\frac{\partial r}{\partial i}\right|\right)+\beta \Psi\left(\left|\frac{\partial g}{\partial i}\right|-\frac{\phi h}{z}\right)\right\}\left(\frac{\partial r}{\partial b_{0}}\right)>0
$$

A equação 30 indica que, pela troca na ordem dos parâmetros no primeiro e no segundo termo, se o primeiro termo for positivo o segundo será provavelmente negativo. Com isso, o primeiro termo da equação 30 deve ser necessariamente positivo para garantir um determinante maior do que zero. Nesse sentido, analisando os parâmetros de políticas econômicas, pode-se concluir que: Quanto menor for a taxa de juros $(\downarrow i)$, maior for o teta $(\uparrow \theta)$ e maior for o beta $(\uparrow \beta)$, maior será o valor 
do determinante, e 2. Quanto maior for o psi $(\uparrow \Psi)$ e o gama $(\uparrow \gamma)$, menor será o determinante.

Para garantir um determinante positivo é necessário, portanto, uma baixa taxa de juros, e que a velocidade de resposta da taxa de juros em relação ao agravamento da fragilidade financeira, ou seja, $\theta \beta>\gamma \Psi$, seja maior do que a do déficit público em relação ao estoque de capital, $\theta>\gamma$. Além do mais, a resposta do déficit público em relação à queda da taxa de lucro, $\beta$, que precede a crise financeira, deve ser elevada. ${ }^{19}$

Portanto, para estabilizar o sistema em um ambiente de severa fragilidade financeira, a coordenação das políticas econômicas - fiscal e monetária - torna-se essencial. Objetivamente, para estabilizar o sistema é necessário que se promova a redução da taxa de juros a níveis bem baixos e que ocorra uma expansão rápida da razão déficit público/estoque de capital em função da queda do lucro agregado.

\section{Considerações Finais}

O modelo proposto destacou que as políticas econômicas devem ser coordenadas, em períodos de severa instabilidade financeira. Apenas uma queda da taxa de juros não torna o sistema estável, ou seja, a flexibilização da política monetária deve ser acompanhada por uma expansão do déficit público, e a resposta da política monetária em decorrência do agravamento da crise financeira deve ser elevada. Destaca-se que o modelo dinâmico também sugere que, em casos de colapso da eficiência marginal do capital, decorrente, por exemplo, de uma deterioração do estado de confiança, a queda dos juros a um nível de "armadilha da liquidez" pode ser requerida para estabilizar a economia, seguida por um aumento do déficit governamental.

O orçamento público torna-se uma peça fundamental no combate ao agravamento das crises financeiras agregadas das empresas privadas, no momento em que a taxa de lucro é reduzida. Nesse sentido, sua administração deve ser responsável, mantendo-se constantemente um orçamento ordinário equilibrado, para, em períodos de dificuldades, utilizar-se do orçamento discricionário de investimento público de forma expansionista. ${ }^{20}$

Severas crises financeiras, acompanhadas de crise fiscal, a qual impossibilita a expansão dos investimentos públicos, ampliação das transferências governamentais e reduções dos impostos certamente tornarão a economia instável por um longo período de tempo.

19 Nessas condições, o modelo apresentará um equilíbrio assintoticamente estável global, de acordo com Takayama (1993, p. 340). Em uma situação de deterioração do estado de confiança, em que a taxa de juros fique muito elevada e $\square$ seja muito baixo, o modelo pode apresentar um ponto de sela com equilíbrio instável (localmente), independentemente do valor do traço da matriz.

Ver Carvalho (1988). 
As ideias centrais de Minsky $(1982,1986,1992)$ vêm corroborando as evidências recentes macroeconômicas. Destacam-se quatro pontos. Primeiro, a questão financeira, que é extremamente relevante para explicar os ciclos de negócio. Segundo, as economias capitalistas, que após a Grande Depressão, não entraram, mas vivenciaram períodos de quedas mais acentuadas da atividade econômica em virtude das atuações mais diretas do Grande Governo e do Grande Banco Central. Terceiro, a atuação do governo, que, em particular, gera os efeitos na demanda agregada, no lucro das empresas e no portfólio das instituições financeiras. Quarto e último, a Grande Depressão, que não foi um fenômeno do acaso; como argumentado por Krugman (2009), as depressões econômicas podem ocorrer novamente ou por ações mal coordenadas de política econômica, falência de grandes instituições bancárias ou colapso da eficácia marginal do capital.

A contribuição deste artigo foi buscar incorporar os aspectos minskyanos em um modelo macroeconômico. E, considerando o pensamento pós-keynesiano, os resultados obtidos corroboram os argumentos de Minsky: o Grande Governo e o Grande Banco são fundamentais para estabilizar a economia em períodos de severa fragilidade financeira. A coordenação das políticas anticíclicas é fundamental e a política monetária deve ser sensível às mudanças severas no estado de confiança. Sistematizar esse pensamento em um modelo matemático foi o principal desafio e contribuição deste trabalho.

\section{Referências}

BORDO, M. Stock market crashes, productivity boom and Bush, and recessions: some historical evidence. Washington, D.C.: International Monetary Fund, 2003.

CARVALHO, F. Keynes, a instabilidade do capitalismo e a teoria dos ciclos. Pesquisa e Planejamento Econômico, Rio de Janeiro, v. 18, n. 3, p. 741-764, dez. 1988.

DAVIDSON, P. Financial markets, money and the real world. Cheltenham, UK and Northampton, MA, USA: Edward Elgar Publishing, 2002.

FISHER, I. The debt-deflation theory of Great Depression. Econometrica, v. 1, n. 4, p. 337-57, Oct. 1933.

FOLEY, D. K. Financial fragility in developing economies. New York, USA: Department of Economics, New School University, 2001.

KEYNES, J. M. A teoria geral do emprego, do juro e da moeda. São Paulo: Nova Cultural, 1996. Publicado originalmente em 1936.

KINDLEBERG, C. Manias, pânico e crashes: um histórico das crises financeiras. Rio de Janeiro: Nova Fronteira, 2000. 
KINDLEBERG, C.; ALIBER, R. Da euforia ao pânico: uma história das crises financeiras. São Paulo: Gente, 2009.

KRUGMAN, P. The return of depression on economics and the crisis of 2008. New York: W. W. Norton $\mathcal{E}$ Company, 2009.

LIMA, G.; MEIRELLES, A. A macrodynamics of debt regimes, financial instability and growth. In: ENCONTRO NACIONAL DE ECONOMIA DA ANPEC, 32., 2004, João Pessoa. Anais... João Pessoa: Anpec, 2004b.

Debt, financial fragility and economic growth: a post-keynesian macromodel. Presented at conference Economic Growth and Distribution: On the Nature and Causes of the Wealth of Nations, Lucca, Italy, June 16, 2004a.

MINSKY, H. P. Stabilizing an unstable economy. New York: McGraw Hill, 1986.

. The financial instability hypothesis. Annandale-on-Hudson, NY: The Jerome Levy Economics Institute of Bard College, May. 1992. (Working Paper, n. 74).

. The financial-instability hypothesis: capitalist process and the behavior of the economy. In: KINDLEBERGER, C. P.; LAFARGUE, J.-P. (Ed.). Financial crises: theory, history, and policy. New York: Cambridge University Press, 1982. p. 13-39.

SORDI, S.; VERCELLI, A. Financial fragility and economic fluctuations: numerical simulations and policy implications. Siena: Univerdità degli Studi di Siena, 2003.

TAKAYAMA, A. Analytical methods in economics. Michigan: University of Michigan Press, 1993.

TAYLOR, L.; O`CONNELL, S. A Minsky crisis. Quarterly Journal of Economics, v. 100, Issues Supplement, p. 871-885, 1985.

VERCELLI, A. Minsky, Keynes and the structural instability of a sophisticated monetary economy. Siena: Univerdità degli Studi di Siena, 1999.

WRAY, L. R. Extending Minsky's classifications of fragility to government and the open economy. Annandale-on-Hudson, NY: The Levy Economics Institute of Bard College, June 2006. (Working Paper, n. 450). 